\title{
Prevalência de anemia em idosos internados em enfermarias gerais de um hospital universitário
}

\author{
Rilva Lopes de Sousa Muñoz*, Graziela Batista de Sousa**, Germana Ribeiro de Lucena**, \\ Mayara Cardoso Grigório*, Leina Yukari Etto***
}

\section{Resumo}

A anemia representa um problema de saúde pública para a população idosa, pois atua negativamente, sobretudo no contexto de hospitalização. Sua prevalência não está clara na literatura em virtude de resultados discrepantes. Os objetivos deste estudo foram avaliar a prevalência de anemia em idosos internados em enfermarias de clínica médica de um hospital universitário brasileiro, assim como determinar a classificação morfológica da anemia e sua distribuiçãa de acordo com a faixa de idade e o sexo. Realizou-se um estudo observacional e transversal envolvendo amostra de cem pacientes com 60 anos ou mais, que se encontravam internados no período. O padrão de referência para diagnóstico de anemia foi o nível de hemoglobina $(\mathrm{Hb})$ abaixo de $12 \mathrm{~g} / \mathrm{dL}$ para muIheres e $13 \mathrm{~g} / \mathrm{dL}$ para homens. Anemia leve foi considerada entre esses valores e $10 \mathrm{~g} /$ dL. A classificação morfológica da anemia foi feita por meio do volume corpuscular médio, da concentração de hemoglobina corpuscular média e do índice de anisocitose eritrocitária. A média de idade foi 70,7 $( \pm 7,2)$ anos, e $54 \%$ da amostra era do sexo masculino. A prevalência de anemia foi de $84 \%$, entre ambos os sexos, normocítica e normocrômica em $63 \%$ dos casos, $41,6 \%$ leve e $27,4 \%$ com anisocitose. Não houve diferença na prevalência de anemia quanto à idade e ao sexo. Conclui-se que a prevalência de anemia em idosos internados em enfermarias clínicas gerais foi elevada, sendo mais frequente a anemia leve e normocítica.

Palavras-chave: Anemia. Hospitalização. Idoso.

\section{Introdução}

Anemia em pacientes idosos é um emergente problema de saúde pública no século 21 (BARBOSA; ARRUDA; DINIZ, 2006; VANASSE; BERLINER, 2010; RACHOIN et al., 2013; GEISEL et al., 2014). Anemia em indivíduos mais velhos está associada a uma ampla gama de complicações, sobretudo no contexto de uma hospitalização: aumento da

* Médica generalista. Professora de Semiologia Médica, Departamento de Medicina Interna, Centro de Ciências Médicas, Universidade Federal da Paraíba, campus I. João Pessoa, Paraíba. Departamento de Medicina Interna, Centro de Ciências Médicas, Universidade Federal da Paraíba, campus I, s. nº , Cidade Universitária, João Pessoa, Paraíba, CEP 58050-000. E-mail: rilva@ccm.ufpb.br / rilvalopes@hotmail.com

* Acadêmicos de Medicina na Universidade Federal da Paraíba, campus I, João Pessoa, Paraíba, Brasil.

**** Médica hematologista. Professora de Semiologia Médica, Departamento de Medicina Interna, Centro de Ciências Médicas, Universidade Federal da Paraíba, campus I. E-mail: leina.etto@gmail.com

$\rightarrow$ http://dx.doi.org/10.5335/rbceh.v13i1.5424

Recebido em: 29/09/2015. Aceito em: 20/05/2016. 
mortalidade, agravamento de doenças preexistentes, complicações cardiovasculares, disfunção cognitiva, incapacidade funcional, hospitalização prolongada e queda significativa na qualidade de vida (EISENSTAEDT; PENNINX; WOODMAN, 2006; BOSCO et al., 2013). $\mathrm{O}$ aumento da mortalidade em idosos ocorre mesmo quando a anemia é leve (ENDRES et al., 2009).

Anemia consiste em uma concentração de hemoglobina sérica anormalmente baixa em consequência da carência de um ou mais nutrientes essenciais, qualquer que seja a origem dessa carência (WORLD HEALTH ORGANIZATION, 2004). Trata-se de uma preocupação comum na subárea de saúde geriátrica, mas sua prevalência não está clara, pois vários estudos mostram resultados discrepantes, com uma variação de 5,5\% a 62,6\% em idosos (MILAGRES et al., 2015).

Os objetivos deste estudo são avaliar a prevalência de anemia em idosos internados nas enfermarias de clínica médica de um hospital universitário brasileiro bem como determinar a classificação morfológica da anemia e sua distribuição de acordo com o sexo e a idade.

\section{Métodos}

Realizou-se um estudo observacional do tipo transversal, envolvendo pacientes com idade maior do que 60 anos, internados consecutivamente nas enfermarias de clínica médica do Hospital Universitário Lauro Wanderley, na cidade de João Pessoa, estado da Paraíba, Brasil, entre julho de 2014 e fevereiro de 2015 .
A amostragem foi não probabilística e intencional, compreendendo cem pacientes de ambos os sexos. O tamanho da amostra baseou-se em estudos anteriores de prevalência de anemia em idosos hospitalizados (BHASIN; RAO, 2011; ZILINSKI et al., 2014). Foram excluídos do estudo os pacientes transfundidos na atual internação. Não houve perdas, todos os pacientes abordados aceitaram participar do estudo e assinaram o termo de consentimento livre e esclarecido.

A coleta de sangue foi realizada entre 8 e 10 horas, com a obtenção de $5,0 \mathrm{~mL}$ de sangue por punção venosa braquial. As amostras foram encaminhadas ao laboratório de análises clínicas do mesmo hospital para as análises hematimétricas, em contador eletrônico de células (Sismex SF 3000, Automated Hematology Analyser, GMI, Inc. Ramsey, MN, USA).

O padrão de referência para diagnóstico de anemia foi o nível de hemoglobina ( $\mathrm{Hb})$ determinado pelo laboratório de análises clínicas do hospital. Foi determinada a prevalência de anemia na amostra com base no nível de $\mathrm{Hb}$ abaixo de $12 \mathrm{~g} /$ $\mathrm{dL}$ para mulheres e $13 \mathrm{~g} / \mathrm{dL}$ para homens (WORLD HEALTH ORGANIZATION, 2004). Idosos anêmicos foram classificados como casos graves, quando apresentaram $\mathrm{Hb}$ abaixo de $7 \mathrm{~g} / \mathrm{dL}$ (para ambos os sexos), ou moderados, quando apresentaram $\mathrm{Hb}$ entre $7,1 \mathrm{~g} / \mathrm{dL}$ e $10 \mathrm{~g} / \mathrm{dL}$, para o sexo feminino, e $7,1 \mathrm{~g} / \mathrm{dL}$ e $12 \mathrm{~g} / \mathrm{dL}$, para o masculino. Anemia leve foi considerada entre $12 \mathrm{~g} / \mathrm{dL}$ ou $13 \mathrm{~g} / \mathrm{dL}$ e $10 \mathrm{~g} / \mathrm{dL}$ (MILAGRES et al., 2015). A classificação morfológica da anemia foi feita por meio do volume corpuscular médio, 
da concentração da hemoglobina corpuscular média e do índice de anisocitose eritrocitária, cujos parâmetros normais de referência foram os recomendados pela Organização mundial da Saúde (OMS) (Quadro 1).

Quadro 1 - Valores de referência dos parâmetros hematimétricos segundo o sexo

\begin{tabular}{l|r|r}
\hline \multicolumn{1}{c|}{ Parâmetros } & \multicolumn{1}{c|}{ Mulheres } & \multicolumn{1}{c}{ Homens } \\
\hline $\begin{array}{l}\text { Hemoglobina (g/dL) } \\
\text { Volume corpuscular } \\
\text { médio (fL) }\end{array}$ & 812,0 & $<13,0$ \\
$\begin{array}{l}\text { Concentração } \\
\text { da hemoglobina } \\
\text { corpuscular média (\%) } \\
\begin{array}{l}\text { Índice de anisocitose } \\
\text { eritrocitária (\%) }\end{array}\end{array}$ & $32,0-35,8$ & $80,0-98,0$ \\
\hline
\end{tabular}

Fonte: de acordo com Barbosa, Arruda e Diniz (2006).

Os diagnósticos principais que motivaram a internação foram agrupados conforme a Classificação Estatística Internacional de Doenças e Problemas Relacionados à Saúde - CID-10 (ORGANIZAÇÃO MUNDIAL DA SAÚDE, 2014). Foram incluídos tanto pacientes com anemias primárias quanto secundárias a outras patologias.

$\mathrm{Na}$ estatística descritiva, foram determinadas as frequências relativas e absolutas referentes às variáveis qualitativas, assim como as médias e os desvios-padrão das variáveis quantitativas. Na estatística inferencial, foram usados os testes de Mann-Whitney e de Kruskall-Wallis para as variáveis ordinais e intervalares. A significância estatística adotada foi de 5\%. Empregou-se o programa estatístico SPSS 20.0 para Windows nas análises.
O projeto desta pesquisa foi aprovado pelo Comitê de Ética em Pesquisa do hospital onde foi realizado o estudo, com parecer sob registro CAAE 43731615.50000.5183, seguindo o que normatiza a Resolução 466/2012, do Conselho Nacional de Saúde e do Ministério da Saúde.

\section{Resultados}

Avaliaram-se cem idosos consecutivamente internados nas enfermarias de clínica médica do Hospital Universitário Lauro Wanderley, com idades entre $60 \mathrm{e}$ 91 anos, $52 \%$ com 60 a 69 anos, $34 \%$ de 70 a 79 e $14 \%$ de 80 anos ou mais. A caracterização demográfica está demonstrada na Tabela 1.

Tabela 1 - Características demográficas dos pacientes idosos internados nas enfermarias de clínica médica do Hospital Universitário Lauro Wanderley $(n=100)$ entre 2014 e 2015

\begin{tabular}{|c|c|}
\hline Variáveis & Estatísticas \\
\hline Sexo (\% masculino) & 46 \\
\hline Sexo (\% feminino) & 54 \\
\hline Estado civil (\% casados) & 58 \\
\hline Procedência (\% capital) & $62 \%$ \\
\hline Idade (anos, M \pm DP) & $70,7( \pm 7,2)$ \\
\hline Cor (\% pretos/pardos) & 66 \\
\hline \multicolumn{2}{|l|}{ Escolaridade (\%) } \\
\hline Analfabetos & 32 \\
\hline Alfabetizados & 9 \\
\hline Fundamental incompleto & 21 \\
\hline Fundamental completo & 8 \\
\hline Médio incompleto & 13 \\
\hline Médio completo & 6 \\
\hline Sem informação & 11 \\
\hline
\end{tabular}

Fonte: primária.

Nota: M: Médias; DP: desvio padrão. 
Verificou-se que 57\% das internações foram motivadas por doenças dos aparelhos circulatório, digestivo e respiratório, as quais foram seguidas pelas motivadas por doenças hematológicas, responsáveis por $12 \%$ das hospitalizações (Tabela 2).

Tabela 2 - Distribuição dos idosos internados nas enfermarias de clínicas médica e cirúrgica do Hospital Universitário Lauro Wanderley $(\mathrm{n}=100)$ entre 2014 e 2015 de acordo com o diagnóstico de internação conforme a CID-10

\begin{tabular}{l|c}
\hline $\begin{array}{l}\text { Classificação Internacional de Doenças } \\
\text { (CID- 10) }\end{array}$ & $\begin{array}{c}\text { Frequências } \\
f / \%\end{array}$ \\
\hline $\begin{array}{l}\text { Doenças do aparelho circulatório } \\
\text { Doenças do sistema digestivo }\end{array}$ & 21 \\
Doenças do aparelho respiratório & 21 \\
Doenças do sangue e órgãos & 15 \\
hematopoiéticos & 12 \\
$\begin{array}{l}\text { Doenças do aparelho geniturinário } \\
\text { Diagnóstico não definido }\end{array}$ & 7 \\
$\begin{array}{l}\text { Doenças do tecidos osteomuscular } \\
\text { e conjuntivo }\end{array}$ & 7 \\
$\begin{array}{l}\text { Doença endócrinas, nutricionais e } \\
\text { metabólicas }\end{array}$ & 6 \\
Doenças infecciosas e parasitárias & 4 \\
$\begin{array}{l}\text { Doenças do sistema nervoso } \\
\text { Neoplasias }\end{array}$ & 4 \\
Transtornos mentais e & 1 \\
comportamentais & 1 \\
\hline
\end{tabular}

Fonte: primária.

A prevalência de anemia na amostra foi de $84 \%$, entre ambos os sexos, em $63 \%$ dos casos anemia normocítica e normocrômica, $27,4 \%$ com anisocitose e $22,6 \%$ como anemia grave (Tabela 3). A análise dos dados hematimétricos revelou ainda que anisocitose com microcitose estava presente em $14,3 \%(12 / 84)$ e isocitose com normocromia em $52,4 \%$ (44/84) dos idosos anêmicos.
Tabela 3 - Classificação dos casos de anemia em idosos internados nas enfermarias de clínica médica do Hospital Universitário Lauro Wanderley de acordo com o sexo $(n=84)$

\begin{tabular}{|c|c|c|}
\hline \multirow{2}{*}{ Classificação da anemia } & \multicolumn{2}{|c|}{ Frequências } \\
\hline & $f$ & $\%$ \\
\hline \multicolumn{3}{|l|}{ Intensidade } \\
\hline Leve & 35 & 41,6 \\
\hline Moderada & 30 & 35,7 \\
\hline Grave & 19 & 22,6 \\
\hline \multicolumn{3}{|l|}{ Morfologia (VCM/CHCM) } \\
\hline Normocítica/normocrômica & 53 & 63,0 \\
\hline Microcítica/hipocrômica & 15 & 17,9 \\
\hline Microcítica/normocrômica & 14 & 16,7 \\
\hline Macrocítica & 2 & 2,4 \\
\hline \multicolumn{3}{|l|}{$\begin{array}{l}\text { Morfologia (variação de } \\
\text { tamanho das hemácias, RDW) }\end{array}$} \\
\hline Isocitose & 61 & 72,6 \\
\hline Anisocitose & 23 & 27,4 \\
\hline \multicolumn{3}{|l|}{ Fonte: primária. } \\
\hline $\begin{aligned} \text { Nota: } & \text { VCM: volume corpuscular me } \\
& \text { ção da hemoglobina corpuscul } \\
& \text { distribution width (índice de a }\end{aligned}$ & $\mathrm{ICN}$ & entra \\
\hline
\end{tabular}

A presença de anemia não diferiu entre homens e mulheres ( $p=\mathrm{NS}$ ) como mostra a Figura 1. Verificou-se que $81,5 \%$ dos homens e $86,9 \%$ das mulheres da amostra apresentavam anemia. Também não houve diferença estatisticamente significativa nas idades entre os pacientes anêmicos e não anêmicos (Figura 2), observando-se médias e medianas superiores nos pacientes sem anemia, porém sem diferença estatisticamente significativa em relação aos pacientes anêmicos. 
Figura 1 - Distribuição percentual dos casos de anemia e sua intensidade em idosos internados nas enfermarias de clínicas médica do Hospital Universitário Lauro Wanderley, de acordo com o sexo $(n=84)$
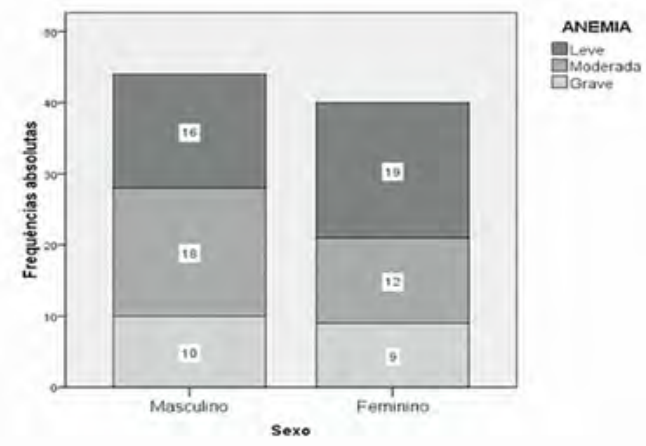

Fonte: coleta de dados da pesquisa.

Figura 2 - Comparação entre as idades dos pacientes anêmicos e não anêmicos em amostra de cem idosos internados nas enfermarias de clínicas médica do Hospital Universitário Lauro Wanderley, de acordo com o sexo $(\mathrm{n}=100)$

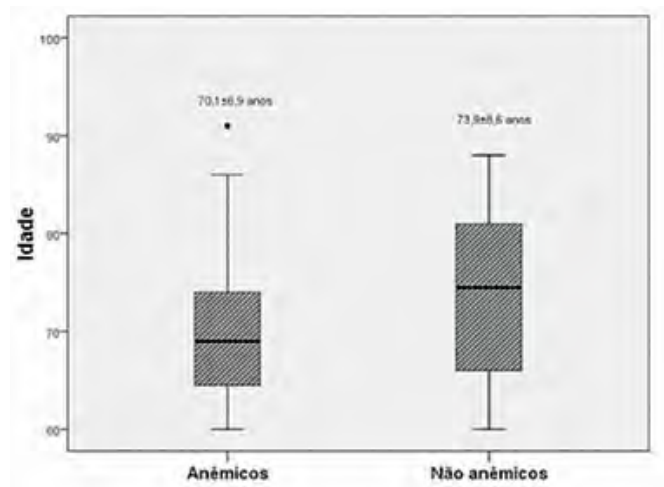

Fonte: coleta de dados da pesquisa.

Dos 52 idosos de 60 a 69 anos, de ambos os sexos, 45 apresentavam anemia $(86,5 \%)$, enquanto entre os 34 pacientes de 70 a 79, 28 estavam anêmicos $(85,3 \%)$ e 10 dos 14 com 80 anos ou mais $(71,4 \%)$ apresentavam essa condição. Entre os 84 pacientes com anemia, $56(66,6 \%)$ eram pardos ou pretos, mas não houve diferença na distribuição de frequências entre esses e os pacientes brancos.

\section{Discussão}

A constatação mais marcante dos resultados do presente estudo é a alta prevalência de anemia em pacientes idosos internados nas enfermarias clínicas do Hospital Universitário Lauro Wanderley, com frequência superior às encontradas na literatura, embora apenas $12 \%$ das internações da amostra deste estudo tenham sido motivadas por doenças hematológicas. Apesar de uma revisão sistemática revelar que anemia teve prevalência, variando entre $40 \%$ e $72 \%$ em quatro estudos, totalizando um contingente de 13.953 idosos hospitalizados (GASKELL et al., 2008), essas são prevalências inferiores à encontrada neste estudo (86,1\%). Resultados de outra revisão sistemática indicam que em estudos com idosos hospitalizados, as prevalências de anemia foram $67,5 \%$, $55 \%, 46,3 \%$ e $36,7 \%$ (MILAGRES et al., 2015).

Estudo retrospectivo com 812 pacientes de 65 anos ou mais internados em enfermarias de clínica médica do Hospital Universitário de Singapura demonstrou que a anemia estava presente em $57,1 \%$ dos casos (TAY; ONG, 2011). Em estudo retrospectivo de 405 pacientes geriátricos hospitalizados na Alemanha $(74,8 \%$ homens e $62,9 \%$ mulheres), $66,3 \%$ esta- 
vam anêmicos de acordo com os critérios da OMS, a maioria $(85,1 \%)$ com anemia leve (GEISEL et al., 2014). Em estudo prospectivo também em pacientes idosos com 70 anos ou mais hospitalizados na Alemanha ( $\mathrm{n}=100)$, em enfermarias clínicas, a prevalência de anemia foi de $60 \%$ (ZILINSKI et al., 2014). Em estudo realizado com 6.880 idosos, sem comorbidades graves, demonstrou-se que anemia leve foi encontrada em $6,1 \%$ das mulheres e $8,1 \%$ dos homens (ENDRES et al., 2009). Esses resultados são expressivamente inferiores aos apresentados neste estudo e também aos dos outros mencionados anteriormente. Um estudo no Brasil evidenciou que de 709 pacientes idosos com idades de 60 anos ou mais internados em um hospital de Belo Horizonte, Minas Gerais, 30\% estavam com anemia (BOSCO et al., 2013), prevalência também marcadamente inferior à encontrada nesta pesquisa.

Estudos realizados com idosos hospitalizados em outros países também apresentam prevalências inferiores aos encontrados no presente estudo. Cem pacientes com idade acima de 60 anos (média de 70,5 anos; $52 \%$ do sexo masculino), hospitalizados em um hospital na Índia, apresentavam anemia em 68\% dos casos (BHASIN; RAO, 2011).

Pacientes idosos com problemas de saúde suficientemente graves para motivar hospitalização são mais frequentemente afetados por anemia do que a população geriátrica como um todo, pois apresentam mais frequentemente infecção aguda, comorbidades, doenças graves e um maior risco de hemorragias
(GEISEL et al., 2014). De outro modo, há evidências que sugerem que as células-tronco hematopoiéticas exibem crescente resistência à eritropoetina com 0 aumento da idade, e, também, que o envelhecimento está associado ao aumento da expressão de citocinas pró-inflamatórias, que contribuiriam para a indução de anemia. Estudos recentes sugerem que, embora a anemia, provavelmente, surja, em parte, pelo efeito cumulativo de comorbidades relacionadas com a idade e o declínio físico, as referidas mudanças específicas pela idade no sistema hematopoiético influenciam fortemente a produção de hemácias (VANASSE; BERLINER, 2010).

Contudo, as consideráveis diferenças de prevalência encontradas na literatura e nos resultados desta pesquisa podem ser devidas às variações na média de idade das amostras/populações em estudo, às diferenças no número de comorbidades presentes e à gravidade dos próprios diagnósticos principais. A variabilidade na prevalência entre diversos estudos pode estar também relacionada aos critérios utilizados para definir anemia. Os critérios estabelecidos pela Organização Mundial de Saúde - nível de hemoglobina menor que $12 \mathrm{~g} / \mathrm{dL}$ para mulheres e menor que $13 \mathrm{~g} / \mathrm{dL}$ para homens - foram usados em todos os estudos comparáveis publicados e referidos anteriormente. Contudo, a adequação desses parâmetros a populações mais velhas tem sido questionada (BEGHÉ; WILSON; ERSHLER, 2004). Embora especialistas tenham sugerido a substituição dos padrões da OMS por definições baseadas em estudos mais recentes, envolvendo idosos, 
critérios revisados ainda não foram adotados (GUALANDRO; HOJAIJ; JACOB FILHO, 2010).

$\mathrm{O}$ achado de maior frequência masculina de anemia em idosos hospitalizados reportado em estudos anteriores (GEISEL et al., 2014; ENDRES et al., 2009; GURALNIK et al., 2004) não foi corroborado neste estudo. Alguns autores relatam um aumento particularmente notável na prevalência de anemia em indivíduos mais velhos, aqueles com idade igual ou maior que 85 anos de idade (BEGHÉ; WILSON; ERSHLER, 2004). Esse resultado discrepante da associação de anemia com o aumento da idade pode referir-se à população idosa incluída neste estudo, uma vez que são pacientes com problemas grave de saúde (e presumivelmente, com risco acrescido de anemia), o que pode ter influenciado o resultado de a prevalência de anemia não aumentar com a idade, pois essa relação é reportada na população geriátrica como um todo, abrangendo idosos vivendo na comunidade, fora de contextos clínicos e em atendimento ambulatorial. De forma similar ao que foi observado nesta amostra, no estudo de Geisel et al. (2014), também não se encontrou associação entre anemia e aumento da idade em idosos hospitalizados.

A alta frequência de comorbidades em pacientes idosos parece ser um fator de confusão para o estabelecimento de uma tendência para a redução da hemoglobina relacionada à idade, pois mesmo em indivíduos saudáveis, a incidência é muito maior em pacientes com múltiplas comorbidades. Vanasse e Berliner (2010) postulam que a tendência a um estado pró-inflamatório na população idosa predispõe à sua acentuada debilidade face à presença de comorbidades.

A anemia normocítica e normocrômica também foi a mais frequente em outros estudos com idosos hospitalizados (GEISEL et al., 2014; ENDRES et al., 2009; GURALNIK et al., 2004; BHASIN; RAO, 2011). Essa classificação morfológica pode indicar a suposta etiologia de anemia da doença crônica como causa plausível. Além disso, a anemia de doença crônica é considerada a causa mais frequente de anemia em pacientes adultos hospitalizados (MATOS et al., 2008).

Pelo fato de a anemia ser um sinal, e não um diagnóstico etiológico, uma avaliação da causa é sempre necessária diante dessa condição. Poucos estudos têm investigado o perfil etiológico da anemia em pacientes hospitalizados na faixa etária idosa (GEISEL et al., 2014; JOOSTEN et al., 1992). Em estudo anterior, a anemia foi atribuída, principalmente, à doença crônica subjacente $(62,1 \%)$, ou de etiologia mista, envolvendo uma combinação de doença crônica e deficiência de ferro (GEISEL et al., 2014). As etiologias da anemia devem ser determinadas em todos os casos, a fim de facilitar a escolha e a implementação de uma terapia eficaz, o que pode ser visto como uma limitação do presente estudo, pois as causas das anemias não foram avaliadas. No entanto, o objetivo desta investigação foi deliberadamente o de avaliar a prevalência de anemia em pacientes geriátricos admitidos nas enfermarias clínicas do Hospital Univer- 
sitário Lauro Wanderley, para avaliar a magnitude do problema, independentemente de sua etiologia.

A aplicabilidade prática desse resultado volta-se à sua implicação clínica para essa clientela e deve ser objeto de discussão médica nas enfermarias clínicas do hospital. O reconhecimento da anemia nos idosos é importante por várias razões, entre elas, porque a anemia pode representar o primeiro sinal de uma doença grave subjacente, tal como câncer do trato digestivo ou deficiência de vitamina B12, que pode ser letal, se não diagnosticada, além do fato de que, por si mesma, a anemia está associada à maior dependência funcional (BOSCO et al., 2013), demência, insuficiência cardíaca, aumento do risco de reações terapêuticas adversas e maior mortalidade (BALDUCCI, 2010).

\section{Conclusões}

A prevalência de anemia em idosos internados em enfermarias clínicas do Hospital Universitário Lauro Wanderley foi muito elevada, superior às prevalências publicadas na literatura brasileira e internacional, e apresentou-se mais frequentemente como anemia leve e normocítica, sem diferença quanto ao sexo e ao aumento da idade. Maior atenção para anemia em idosos hospitalizados é necessária devido à sua alta prevalência e ao provável impacto negativo sobre os desfechos clínicos dos pacientes. Os idosos hospitalizados devem ser examinados rotineiramente em relação à possibilidade de anemia.

\section{Prevalence of anemia among elderly in patients of general wards of a university hospital}

\section{Abstract}

Anemia is a public health problem in the elderly, in which acts negatively, especially in the context of a hospital, but their prevalence is not clear in the literature because of conflicting results. The objectives of this study were to assess the prevalence of anemia in elderly patients in medical clinics wards at a university hospital of Brazil, and to determine the morphological classification of anemia and its distribution according to age group and gender. An observational and cross-sectional study was realized including a sample of 100 patients aged 60 or more admitted to the medical wards. The reference standard for diagnosis of anemia was the level of hemoglobin $(\mathrm{Hb})$ as below $12 \mathrm{~g} / \mathrm{dL}$ for women and $13 \mathrm{~g} / \mathrm{dL}$ for men. Mild anemia was seen between these values and $10 \mathrm{~g} / \mathrm{dL}$. The morphological classification of anemia was made through the mean corpuscular volume, mean corpuscular hemoglobin concentration and erythrocyte anisocytosis index. The average age was 70,7 $( \pm 7,2)$ years, and $54 \%$ were men. The prevalence of anemia was $84 \%$, normocytic and normochromic in $63 \%$ of cases, $41,6 \%$ and $27,4 \%$ with mild anisocytosis. There was no difference in the prevalence of anemia as age and sex. We conclude that the prevalence of anemia in elderly patients admitted to the general medical wards is high, it presents most often as mild and normocytic.

Keywords: Anemia. Hospitalization. Elderly. 


\section{Referências}

BALDUCCI, L. Anemia, cancer, and aging. Cancer Control, v. 10, n. 6, p. 479-486, 2003.

BARBOSA, D. L.; ARRUDA, I. K. G.; DINIZ, A. S. Prevalência e caracterização da anemia em idosos do Programa de Saúde da Família. Revista Brasileira de Hematologia e Hemoterapia, São José do Rio Preto, v. 28, n. 4, p. 288-292, 2006.

BEGHÉ, C.; WILSON, A.; ERSHLER, W. B. Prevalence and outcomes of anemia in geriatrics: a systematic review of the literature. American Journal of Medicine, v. 116, Suppl 7A, p. 3S-10S, 2004.

BHASIN, A.; RAO, M. Y. Characteristics of anemia in elderly: a hospital based study in south India. Indian Journal of Hematology \& Blood Transfusion, v. 27, n. 1, p. 26-32, 2011.

BOSCO, R. M. et al. Anemia and functional capacity in elderly Brazilian hospitalized patients. Cadernos de Saúde Pública, Rio de Janeiro, v. 29, n. 7, p. 1.322-1.332, 2013.

EISENSTAEDT, R.; PENNINX, B. W.; WOODMAN, R. C. Anemia in the elderly: current understanding and emerging concepts. Blood Reviews, v. 20, n. 4, p. 213-226, 2006.

ENDRES, H. G. et al. Prevalencee of anemia in elderly patients in primary care: impact on 5-year mortality risk and differences between men and women. Current Medical Research and Opinion, v. 25, n. 5, p. 1.1431.158, 2009.

GASKELL, H. et al. Prevalence of anaemia in older persons: systematic review. BMC Geriatrics, v. 8, n. 1, não paginado, 2008. Disponível em: <http://www.ncbi.nlm.nih. gov/pmc/articles/PMC2248585/>. Acesso em: 14 set. 2015.

GEISEL, T. et al. An etiologic profile of anemia in 405 geriatric patients. Anemia, Cairo, v. 2014, não paginado, 2014. Article Id 932486. Disponível em: <http://www.hin- dawi.com/journals/anemia/2014/932486/>. Acesso em: 14 set. 2015.

GUALANDRO, S. F. M.; HOJAIJ, N. H. S. L.; JACOB FILHO, W. Iron deficiency in the elderly. Revista Brasileira de Hematologia e Hemoterapia, São José do Rio Preto, v. 32, supl. 2, p. 57-61, 2010.

GURALNIK, J. M. et al. Prevalence of anemia in persons 65 years and older in the United States: evidence for a high rate of unexplained anemia. Blood, v. 104, n. 8, p. 2.263-2.268, 2004.

JOOSTEN, E. et al. Prevalence and causes of anaemia in a geriatric hospitalized population. Gerontology, v. 38, n. 1-2, p. 111-117, 1992.

MATOS, J. F. et al. O papel do RDW, da morfologia eritrocitária e de parâmetros plaquetários na diferenciação entre anemias microcíticas e hipocrômicas. Revista Brasileira de Hematologia e Hemoterapia, São José do Rio Preto, v. 30, n. 6, p. 463-469, 2008.

MILAGRES, C. S. et al. Prevalência e etiologia da anemia em idosos: uma revisão integral. Medicina Ribeirao Preto (On-line), Ribeirão Preto, v. 48, n. 1, p. 99-107, 2015. Disponível em: <http://www.revistas.usp. br/rmrp/article/view/97023>. Acesso em: 28 ago. 2015.

ORGANIZAÇÃO MUNDIAL DA SAÚDE. Classificação estatística internacional de doenças e problemas relacionados à saúde - CID-10. 10. ed. São Paulo: EDUSP, 2014.

RACHOIN, J. S. et al. Prevalence and impact of anemia in hospitalized patients. Southern Medical Journal, v. 106, n. 3, p. 202-206, 2013.

VANASSE, G. J.; BERLINER, N. Anemia in elderly patients: an emerging problem for the 21st century. Hematology American Society Hematology Education Program, v. 2010, n. 1, p. 271-275, 2010. 
TAY, M. R. J.; ONG, Y. Y. Prevalence and risk factors of anaemia in older hospitalised patients. Proceedings of Singapore Healthcare, v. 20, n. 2, p. 71-79, 2011.

ZILINSKI, J. et al. Prevalence of anemia among elderly inpatients and its association with multidimensional loss of function. Annals of Hematologiy, v. 93, n. 10, p. 1.645-1.654, 2014.

WORLD HEALTH ORGANIZATION. Assessing the iron status of populations. 2004. Disponível em: <http://www.who.int/ nutrition/publications/micronutrients/anaemia_iron_deficiency/9789241596107.pdf>. Acesso em: 20 ago. 2015. 